\title{
A personalized and platform-independent behavior control system for social robots in therapy: development and applications
}

\author{
Hoang-Long Cao, Greet Van de Perre, James Kennedy, Emmanuel Senft, Pablo Gómez Esteban, Albert De Beir, \\ Ramona Simut, Tony Belpaeme, Dirk Lefeber and Bram Vanderborght
}

\begin{abstract}
Social robots have been proven beneficial in different types of healthcare interventions. An ongoing trend is to develop (semi-)autonomous socially assistive robotic systems in healthcare context to improve the level of autonomy and reduce human workload. This paper presents a behavior control system for social robots in therapies with a focus on personalization and platform-independence. This system architecture provides the robot an ability to behave as a personable character, which behaviors are adapted to user profiles and responses during the human-robot interaction. Robot behaviors are designed at abstract levels and can be transferred to different social robot platforms. We adopt the component-based software engineering approach to implement our proposed architecture to allow for the replaceability and reusability of the developed components. We introduce three different experimental scenarios to validate the usability of our system. Results show that the system is potentially applicable to different therapies and social robots. With the component-based approach, the system can serve as a basic framework for researchers to customize and expand the system for their targeted healthcare applications.
\end{abstract}

Index Terms-robot-assisted therapy, supervised autonomy, social robots, personalized behaviors, platform-independent.

\section{INTRODUCTION}

$\mathbf{S}$ OCIAL robots have been developed to provide therapeutic assistance in healthcare for broad populations of users from children to adults e.g. children with autism, diabetes, physical exercises, elderly care [1]-[4]. Robots used in these contexts are required to understand the environment, human intention and performance, and to follow the therapeutic goals to perform meaningful and personalized interaction [5]-[7]. To lessen these challenges, most studies using socially assistive robots in healthcare are restricted to the Wizard of Oz (WoZ) approach [8], [9], in which robots are pre-programmed or remote-controlled by a human operator [3], [10]-[12]. This approach requires a significant amount of manual work to control the robot which deviates the attention of the operator away from the behaviors of the patient and might lead to missing important therapeutical reactions. Therefore, there is an ongoing trend in social robotics toward developing (semi)autonomous behavior control systems to increase the level

H.-L. Cao, G. Van de Perre, P. Gómez Esteban, A. De Beir, D. Lefeber, and B. Vanderborght are with Robotics and Multibody Mechanics Research Group, Vrije Universiteit Brussel and Flanders Make, Brussels, Belgium.

R. Simut is with the Department of Clinical and Life Span Psychology, Vrije Universiteit Brussel, Brussels, Belgium.

J. Kennedy, E. Senft, and T. Belpaeme are with Centre for Robotics and Neural Systems, Plymouth University, Plymouth, U.K.

Manuscript received $* * *$; revised $* * *$. of robot autonomy and lighten direct human control while fulfilling the desired requirements [7], [11], [12].

The role of personalization in a robot behavior control system is considered important to sustain user's motivation and engagement [5], [6]. Personalization should be considered from both therapeutic perspective (e.g. interaction scenario) and robot behavior perspective to reflect the user's needs, requirements and preferences [13]-[15]. Behaviors can be personalized at different levels from physical level to cognitive and social levels taking into account static (e.g. user name, gender, personality) and dynamic (e.g. emotion, current response) parameters [16], [17]. This points out that personalization should be implemented throughout different parts/modules of the behavior control system.

The role of the therapists in robot assisted therapy systems should not be neglected. Results from surveys investigating the general public as well as the therapists recommend that therapist should not be replaced by robots (fully autonomous) for clinical and ethical concern, as well as to compensate for technical limitations [7], [18]-[23]. This indicates that the robot behavior control system should be organized for the ease of shared-control.

Another issue is that the current behavior control systems used are typically developed for particular therapeutic scenarios and robot platforms [6]. The developed systems and importantly the robot behaviors are not easily applicable and transferable to other applications, which is known as the correspondence problem [24], [25]. Some studies propose that the robot behaviors should be programmed at abstract levels and can be translated to low-level commands for different robot morphologies [6], [7], [12], [26]-[29].

Taking into account the above-addressed issues, we present in this paper a robot behavior control system to generate personalized behaviors under therapist supervision and can be applied to different robot platforms. The system architecture enables the robot to behave as a personable character, which behaviors are adapted to user profiles and responses during the human-robot interaction. Personalized behaviors are generated using a generic method allowing the behaviors to be performed in different robot platforms [28], [29]. The system is implemented following the component-based software engineering (CBSE) approach allowing the developed components to be replaceable or reusable [30]-[33]. We introduce three experimental scenarios in autism therapy, elderly care, and physical exercise to validate the usability of our system. 
Results show that the system is potentially applicable in different therapeutic scenarios. Moreover, with the componentbased approach, the system architecture can serve as a basic framework for researchers to customize and expand the system for their applications.

The paper is organized as follows. Section II overviews the related work on autonomous behavior control systems, personalized behaviors, and generic methods to generate behaviors. Section III presents the approach and design principles. Section IV and V describe the design and implementation of our proposed system. Section VI introduces some case studies to validate the system. Finally, the conclusion is given.

\section{RELATED WORK}

\section{A. Robot Behavior Control Architectures in Therapies}

Robot behavior control architecture is the central part of a socially assistive robotic system allowing the robot control to go beyond the WoZ approach. The architecture enables the robot to select proper behaviors according to external events from the environment and changes of the robot's internal variables. The organization of the architecture is important to the success of a robot-assisted intervention because the success of such intervention hinges on the behavior of the robot [34].

Robot behavior control architectures in therapies have been designed following various approaches to achieve therapeutic goals (see [6] for a review). These approaches include event-driven [35], [36], homeostasis [23], [37] [38], finitestate machine [5] [39], neural networks [40] [41], etc. These architectures enable the robots to generate two main types of behaviors: tasked-based behaviors and social behaviors. Taskbased behaviors are typically generated based on a sequence of robot actions following a certain therapeutic scenario, which can be understandable to the therapists and constrained within specific clinical and ethical boundaries [1], [20]. Social behaviors are generated to enhance the robot's social properties e.g. reaction, attention, emotion, and create the illusion of the robot being alive [42], [43]. Social behaviors are either hard-coded in the task-based actions; or generated, combined/arbitrated by separate modules [6]. To better achieve the therapeutic goal, robot behaviors should sustain user's motivation and engagement during all phases of the therapeutic process by providing personalized behaviors (see Sect. II-B) and interesting play scenarios during and between the interventions [5], [6], [21], [44]-[46].

Previous works (e.g. [6], [7], [12], [18]-[22]) also suggest other important requirements that a robot control architecture in healthcare should acquire:

- Shared-control: Human therapists should not be replaced by robots but supervise the robot operation due to clinical and ethical concerns as well as compensating for technical limitations [6], [7], [18]-[23].

- Scenario-independent and platform-independent: Robot behavior modules developed for one scenario should be reusable for another without significant modifications e.g. reorganizing, re-sequencing [20], [21]. Similarly, robot behaviors should be developed to be expressed in various robot platforms (see Sect.II-C) [6], [7], [12].
- Providing data for analysis: Robot behavior control architectures should record data (e.g. therapeutic performance, user's performance history, robot operation) in structured forms, and also document and analyze the data to reduce the workload of the therapists [6], [21].

\section{B. Personalized Robot Behaviors}

Personalization in social robotics is defined in different but non-conflicting ways. Dautenhahn et al. defined personalization as reflecting the needs and requirements of the social environment where the robot is operating in [14]. Lee et al. described personalization as the creation of a personable character to increase friendliness, to fit user preferences, and to adapt over repeated encounters [15]. Baxter et al. identified three particular facets of personalisation: adaptation of nonverbal behavior, personable language content, and alignment to task performance [13].

These definitions point out that behaviors can be personalized at different levels from physical level to cognitive and social levels taking into account static (e.g. user name, gender, personality) and dynamic (e.g. emotion, current response) parameters [16], [17]. In socially assistive robotic systems, personalization should be considered from therapeutic aspects (e.g. selecting proper interaction scenarios) to robot behavior aspects in both task-based and social behaviors.

\section{Methods for Generating Behaviors for Different Robots}

Robot behaviors especially gestures are often preprogrammed or generated by mapping motion capture data to the robot, which are dependent on the robot's configuration and not easily transferable to other robots [29]. Some studies have attempted to create generic methods allowing the developed robot behaviors to be applied to other robots' morphologies. Stanton et al. used neural networks, which requires training, to flexibly generate gestures for different robots [47]. Others applied techniques used in virtual characters to humanoid robots. Salem et al. abstractly described gestures of MAX virtual agent in three features: the location of the wrist, the shape of the hand and the orientation of the wrist; and translated these features into ASIMO's motor control commands [27]. Similarly, Le et al. used three features: the hand shape, wrist position and palm orientation to generate gestures for NAO robot, which relied on a predetermined table listing all possible wrist positions and the corresponding joint values [26]. Van de Perre et al. used a Body Action Coding System (BACS) to describe abstract human gestures and generate gestures for different robot platforms e.g. NAO, ASIMO, Justin [29]. In this method, a set of joints in a certain robot configuration is considered as a subset of the human body configuration, where virtual joints were added if necessary [29]. Ultimately, these methods reduce hard-coding effort and time to develop and reuse developed robot behaviors.

\section{APPROACH AND DESIGN PRINCIPLES}

\section{A. Approach}

Our approach to design a system architecture was motivated by two fundamental concepts. First, the system should provide 
the robot an ability to behave as a personable character. Second, the developed system can be applied to different therapies and robot platforms. These two concepts are consistent with the requirements derived from related works (Sect. II-A). These requirements function as guidelines for us to form our design principles for the system architecture development. We also use these guidelines as criteria to evaluate the system.

\section{B. Design Principles}

Following the guidelines, we stated three design principles as the basis for the architecture design process. Fig. 1 overviews how the design principles fulfill different elements of the guidelines.

1) Multi-layer behavior: The behavior generation is organized in layers to produce different types of behaviors. We followed the three-layer behavior concept from behavioral psychology, which is widely used in behavioral sciences and robotics [48]-[51]. The behavior system includes the reactive layer, the deliberative layer, and the reflective layer. The reactive and deliberative layers are respectively responsible for generating social behaviors and tasked-based behaviors to follow the therapeutic goals. The reflective layer evaluates the correctness of behaviors generated by the lower layers, and correct them if necessary following the clinical and ethical standards. The reflective layer can be accessed by the therapists allowing shared control.

2) Personalization: The generated behaviors are personalized enabling the robot to behave as a personable character. The architecture should provide the robot an affect system to manage the robot's variables including personality (which can be matched or complementary to the user's personality depending on scenarios [4], [52]-[55]), and affect (mood and emotion). These variables are expressed through the robot behaviors generated by the behavior decision-making.

3) Modularity: The architecture is organized into modules/components for the ease of customization and logging structured data for different parties (e.g. engineers, therapists, patients). The behavior generation is separated with the scenario management to make the system scenario-independent. The behavior generation produces abstract behaviors and is independent to the behavior expression. This allows the generated behaviors to be expressed in different robot platforms. The modularity principle is also applied to the implementation process by using the component-based software engineering approach [30]-[33].

\section{SyStem ARCHITECTURE}

Our proposed system architecture aims to provide a personalized and platform-independent system for robots in different therapies. The system architecture is depicted in Fig. 2. The perceptual system interprets raw data from sensors into abstract interaction events. The affect system allows the robot to behave as a personable character, which is expressed through behaviors generated by a three-layer behavior generation mechanism. These behaviors include tasked-based and social behaviors depending on the therapeutic scenario and user's profile (behavior generation). The behaviors are

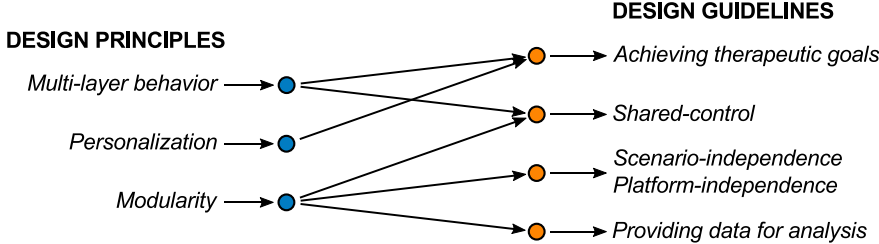

Fig. 1. Relationships between design principles and guidelines derived from related works.

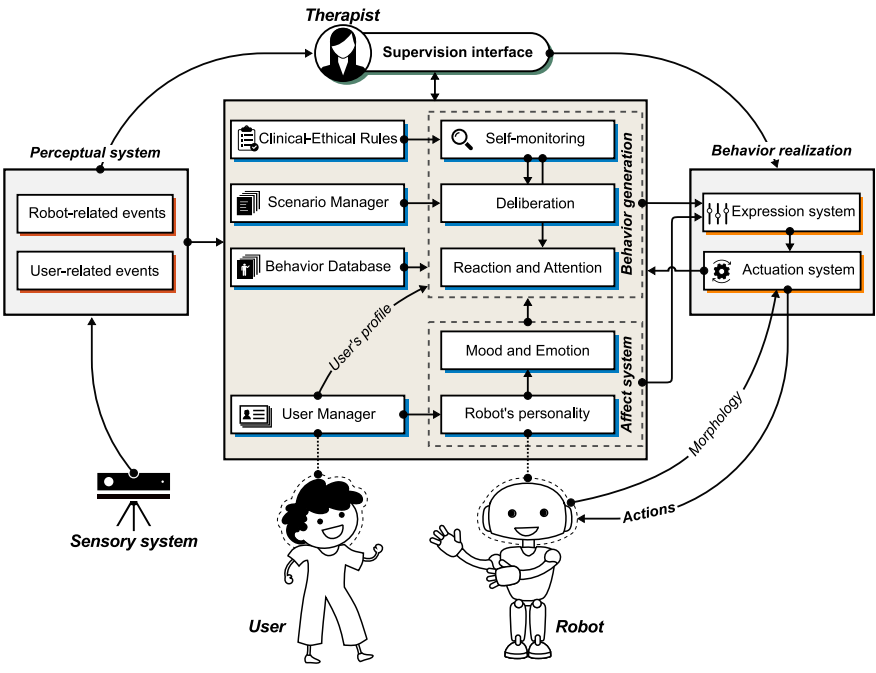

Fig. 2. System architecture. Personalized behaviors are generated taking into account interaction events, user's profile, therapeutic scenario, and clinalethical rules. The abstract behaviors are automatically mapped to different robot platforms. A human therapist supervises the system operation.

accordingly verified automatically by clinical-ethical rules and the therapist. Robot behaviors are designed at abstract levels and translated into robot-special action commands (behavior realization). The following describes general functions of the system elements and models used in each system.

\section{A. Perceptual System}

This system receives raw sensory data from the robot's builtin sensors and external sensors (e.g. cameras) and interprets into abstract interaction events. These events are passed to the behavior generation system and the robot's affect system.

\section{B. User Manager}

This system manages user profiles e.g. name, age, gender, personality, preference, performance history. This data is used to produce personalized behaviors and can be extracted to structured formats for analysis.

\section{Affect System}

This system enables the robot to behave as a personal character by having its own personality and affect (mood and emotion). The robot's personality can be adapted to the user's personality depending on the scenario [4], [52]-[55]. The robot's mood and emotion are changed according to the interaction events. Under the same event, the numerical changes are adapted to the personality. The robot's affect is expressed through the robot's behaviors. 


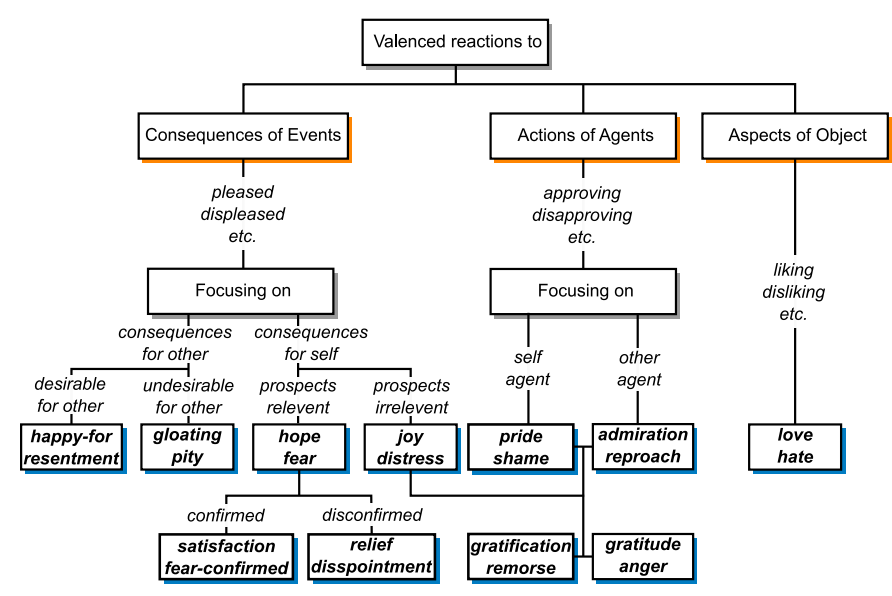

Fig. 3. The OCC model used in the proposed architecture allowing the robot to adapt its emotional state according to events [59].

We applied widely used models in psychology and robotics for the affect system. Personality is modeled by five factors: Openness, Conscientiousness, Extroversion, Agreeableness, and Neuroticism [56], [57]. And affect is modeled by three factors: Valence, Arousal, and Dominance [58].

$$
\mathbf{P}^{T}=\left[\begin{array}{lllll}
O & C & E & A & N
\end{array}\right] \quad \mathbf{E}^{T}=\left[\begin{array}{lll}
V & A & D
\end{array}\right]
$$

These affective values are changed according to the type and intensity of the occurred event, and the robot's personality [60]. These changes are managed by the Orthony Claire Collins model (OCC model) [59] as illustrated in Fig. 3. The model includes 22 basic emotion types (Fig. 4). The mapping of emotion types into the emotion space is based on the work of Gebhard [61], which was adapted from Mehrabian [62]. When there is no event, the robot affect decays to its default value $\mathbf{E}_{\mathbf{0}}$ [61].

$$
\mathbf{E}_{\mathbf{0}}(\mathbf{P})=\left[\begin{array}{c}
V_{0} \\
A_{0} \\
D_{0}
\end{array}\right]=\left[\begin{array}{c}
0.21 E+0.59 A+0.19 N \\
0.15 O+0.30 A-0.57 N \\
0.25 O+0.17 C+0.60 E-0.32 N
\end{array}\right]
$$

When an event $\mathbf{e}_{\mathbf{t}}$ occurs, the OCC model evaluates the event by seven properties i.e. desirability, praiseworthy, appealing, likelihood, liking, realization, and agency [61].

Based on this evaluation, a desired emotion $\mathbf{E}_{\mathbf{t}}^{\mathbf{d}}$ is selected. The robot affect moves from the previous affect position $\mathbf{E}_{\mathbf{t}-\mathbf{1}}$ to the new affect position $\mathbf{E}_{\mathbf{t}}$ taking into account the event intensity calculated from the individual intensities of seven properties $i_{e}\left(\mathbf{e}_{\mathbf{t}}\right)$, and the influence of personality on the desired emotion $i_{P}\left(\mathbf{P}, \mathbf{E}_{\mathbf{t}}^{\mathbf{d}}\right)$.

$$
\mathbf{E}_{\mathbf{t}}=\mathbf{E}_{\mathbf{t}-\mathbf{1}}+i_{e}\left(\mathbf{e}_{\mathbf{t}}\right) \cdot i_{P}\left(\mathbf{P}, \mathbf{E}_{\mathbf{t}}^{\mathbf{d}}\right) \cdot\left(\mathbf{E}_{\mathbf{t}}^{\mathbf{d}}-\mathbf{E}_{\mathbf{t}-\mathbf{1}}\right)
$$

In this work, the event intensity is the greatest value among those of the individual properties [61]. The influences of personality factors on the basic emotion types are summarized in Table I, which is inspired by the review of Doce et al. [63].

\section{Behavior Generation}

This system generates robot abstract behaviors based on user's profiles, interaction events and databases. Following

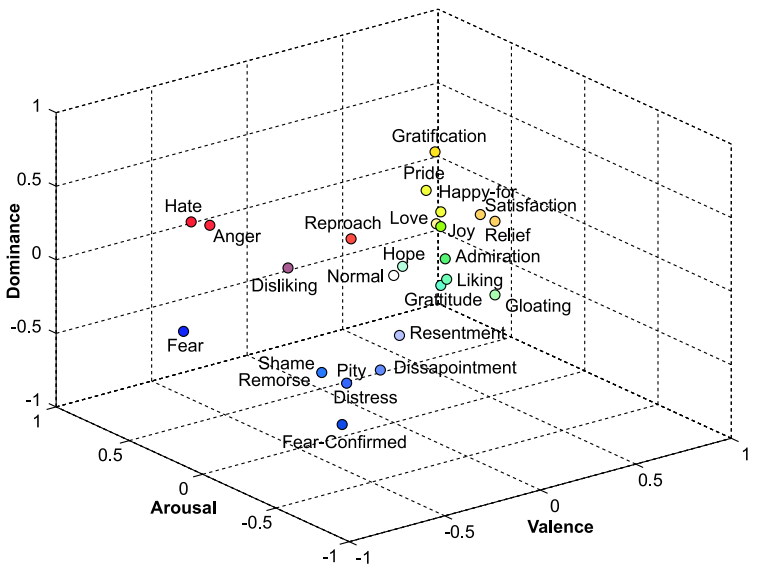

Fig. 4. Positions of OCC basic emotion types in a three-dimensional emotion space [61], [62].

TABLE I

INFLUENCE OF PERSONALITY ON EMOTION TYPES

\begin{tabular}{lll}
\hline Factors & Positive influence & Negative influence \\
\hline Openness & - & $\begin{array}{l}\text { Pride, Shame, Admira- } \\
\text { tion, Reproach }\end{array}$ \\
\hline Conscientiousness & Pride & Gratification \\
\hline Extroversion & - & All emotions \\
\hline Agreeableness & $\begin{array}{l}\text { Love, Happy-For, Pity, } \\
\text { Admiration, Gratitude }\end{array}$ & $\begin{array}{l}\text { Hate, Resentment, Gloat- } \\
\text { ing, Reproach, Anger }\end{array}$ \\
\hline Neuroticism & $\begin{array}{l}\text { Distress, Joy, Disap- } \\
\text { pointment, Relief, Grat- } \\
\text { ification, Fear }\end{array}$ & $\begin{array}{l}\text { Satisfaction, Confirmed, Rears- } \\
\text { Hope }\end{array}$ \\
& & Hemorse, \\
\hline
\end{tabular}

the three-layer behavior organization approach consisting of the reactive layer, the deliberative layer, and the reflective layer [48]-[51], the behavior generation system is composed of three subsystems, namely the reaction and attention, the deliberation, and the self-monitoring subsystems.

The Deliberation subsystem generates task-based behaviors following the current therapeutic scenario, managed by the Scenario Manager. Tasked-based behaviors are personalized by using the user's name and giving feedback based on the user's performance during the interaction.

The Reaction and Attention subsystem generates social behaviors, which creates the illusion of the robot being alive and acts as a catalyst for acceptance [4], [42], [43]. The reaction part includes: falling reaction to avoid physical damage; social reaction to touches and user's emotion; like-life reaction e.g. eye blinking, micro motions. The attention part includes gaze adaptation to the user and sound direction [64].

The Self-monitoring subsystem partially verifies the selected behaviors of the Deliberation and the Reaction and Attention subsystems by referring to Clinical-Ethical Rules. These rules include prohibitions and obligations set by therapists [65]. However, most of the robot behaviors are verified by a human therapist before execution to strictly guarantee the ethical and clinical standards (see IV-E).

\section{E. Therapist Supervision Interface}

This interface provides the therapist an intuitive tool to supervise the robot operation, which is important to increase 
their involvement in the robot's control [66]-[70]. Through this interface, the therapist can verify a generated behavior before execution or select a more proper one if necessary. The technical limitation of the system can be compensated by having the therapist to correct the operation of the perceptual system and the affect system. The interface also provides an ability to switch on/off individual subsystems and manage the databases.

\section{F. Behavior Realization}

This system is responsible for expressing personalized behaviors on the robot platform. It converts abstract generated behaviors and influences of personality and affect into robotspecific behaviors. This process is based on the two following bases.

1) Mapping behavior from human base model to robot configuration: Robot behaviors are designed on a human base model using a Body Action Coding System (BACS). The behavior is converted into robot-specific behavior by adopting a method developed by Van de Perre et al. [28], in which the robot configuration is considered as a subset of a human body configuration, and virtual joints are added if necessary. The joint angles are then calculated using the Runge-Kutte algorithm [71] by the following equation [72]:

$$
\dot{q}=J_{A}^{\dagger}(q)\left(\dot{x_{d}}+K\left(x_{d}-x_{e}\right)\right)+\left(I-J_{A}^{\dagger}(q) J_{A}(q)\right) \dot{q_{0}}
$$

where $x_{d}$ is the desired end effector pose, $J_{A}^{\dagger}(q)$ is the MoorsePenrose pseudo inverse of the analytical jacobian $J_{A}(q), x_{e}$ the current end effector pose, and $\mathrm{K}$ a positive definite gain matrix. Fig. 5 shows an example of happy behavior expressed on different robot platforms.

\begin{tabular}{cccccc}
\hline Human & NAO & Romeo & Pepper & ASIMO & Justin \\
\hline & & & & \\
\hline
\end{tabular}

Fig. 5. Expression of a happy behavior on different robots platforms: virtual models (top) and physical robots (bottom).

2) Influences of personality and affect on behavior expression: Previous works show that valence, arousal, and extroversion have significantly higher influences on behavior expression than other factors [73]-[76]. These factors influence pose's amplitude, palm direction, finger rigidness, head's position, motion speed, holding time, and repetition as suggested by different studies e.g. [73], [74], [77]-[81]. These influences are considered when designing behaviors on the human base model.

The behavior realization process used in our work is illustrated in Fig. 6. In this work, we simplify the influences of valence, arousal, and extroversion into a single affect level formed by the weighted sum of individual factors. Unlike previous works in which affective behaviors are generated based on expression at the neutral affect, our method is based

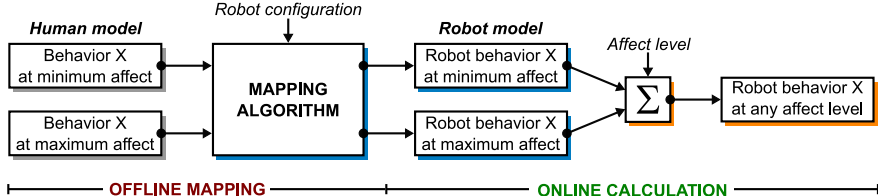

Fig. 6. The process of converting behavior from human base model to robotspecific behavior with affect influence.

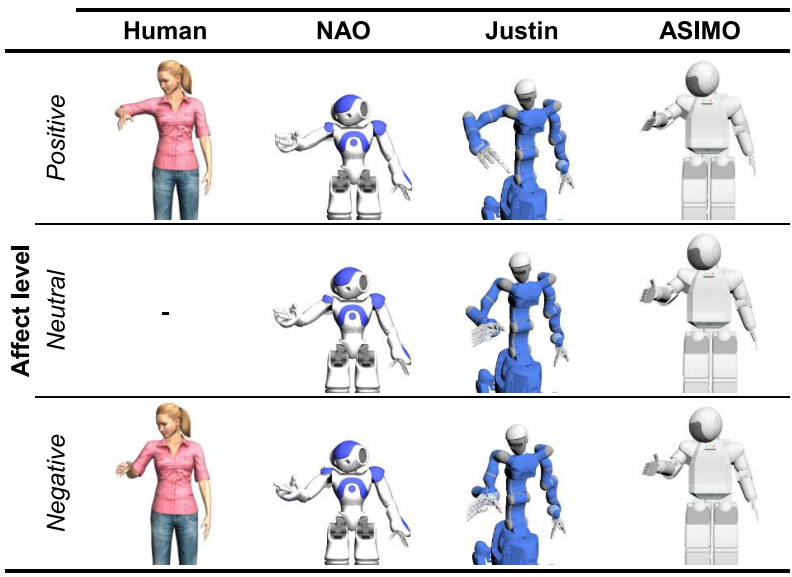

Fig. 7. The end pose of pointing behavior expressed on NAO, Justin, and ASIMO with different affect levels: positive, neutral, negative.

on expression at two extreme affect levels i.e. minimum and maximum.

The process starts from designing behaviors on a humanbased model. Each behavior is coded in two extreme affective levels. This behavior is converted into robot behavior. Behavior expression at a certain affect level is calculated by linear interpolation. The joint angles of each frame of a robot behavior timeline at a certain affect level is calculated by the following equation:

$$
q_{i}=q_{i, \min }+(\underbrace{\alpha_{V} V+\alpha_{A} A+\alpha_{E} E}_{\text {Affect level }})\left(q_{i, \max }-q_{i, \min }\right)
$$

This process does not require inverse kinematics calculation during the robot operation. Hence, computation workload is reduced. An example of this process is illustrated in Fig. 7 showing the end pose of pointing behavior at different affect levels expressed on NAO, Justin, and ASIMO. After this process, the robot-specific robot behavior with calculated joint angles is used to control the physical robot platform.

\section{SYSTEM IMPLEMENTATION}

The system architecture is implemented following the component-based approach [30]-[33] with the aim to increase the reusability and replaceability of developed components (i.e. systems, subsystems). The system is developed using YARP robot developing framework which highly supports component development and system integration [82]-[84].

The implemented system consists of a number of components representing systems and subsystems described in the system architecture (Sect. IV). The communication among components is handled by YARP using ports and connectors. The system is connected to the sensors and robot platform by 


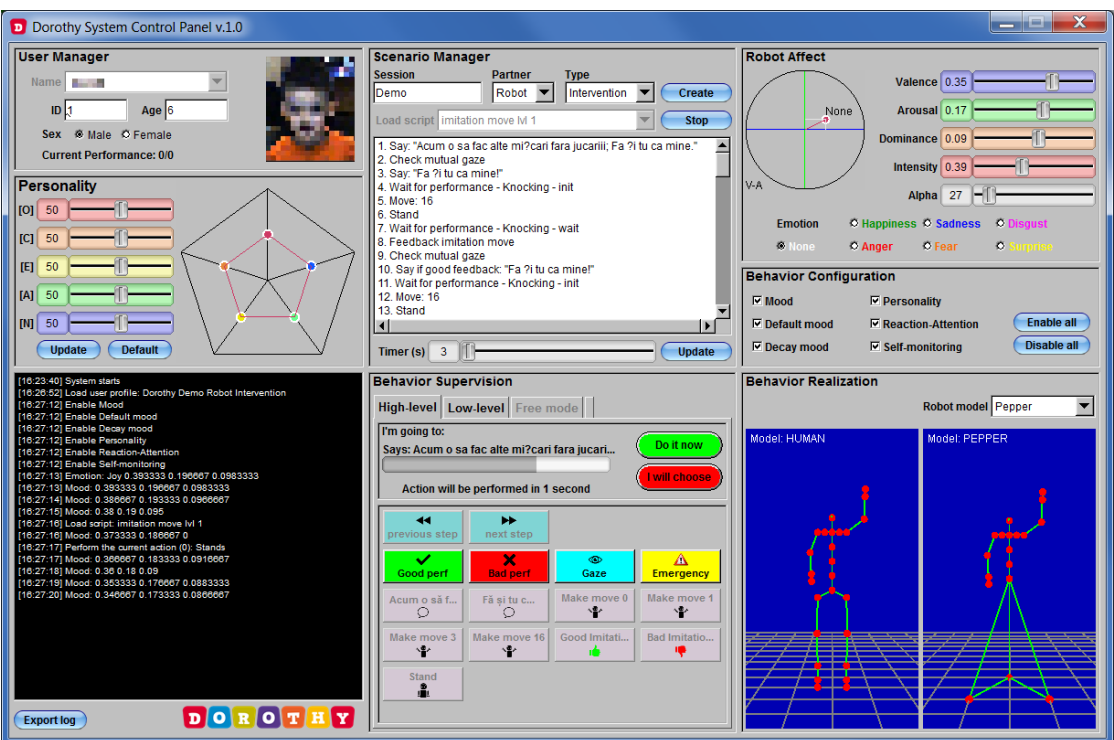

Fig. 8. The system graphical user interface is used to visualize and supervise the system operation. It includes eight group-boxes: User Manager, Scenario Manager, Personality, Robot Affect, Behavior Configuration, Behavior Realization and Log Window. different means of communication depending on the hardware. The system operation is visualized and supervised by a therapist via a graphical-user interface (GUI). The GUI is divided into eight group-boxes as shown in Fig. 8.

User Manager \& Scenario Manager: These group-boxes are used to manage users' profiles, therapeutic scenarios, and to select the interacting user and scenario.

Personality \& Affect: This group-box is used to visualize the robot's personality (which is adapted to user's personality), mood, and emotion. These variables can be manually adjusted by the therapist.

Behavior Supervision: This group-box is the main element for supervision purpose. It allows the therapist to approve or correct autonomously decided behaviors from the behavior generation process (i.e. Approve, Correct, Timeout). Besides that, the therapist can also control the scenario's flow.

Behavior Configuration This group-box is used to switch on/off individual layers of behavior generation and personalization depending on the therapeutic requirements.

Behavior Realization This group-box is used to visualize the personalized behavior on the selected robot platform.

Log window This control box records the system operation and can export data to a structured format for analysis.

\section{System VALIDATION}

In this section, we introduce three experimental scenarios for system validation purpose: autism therapy, elderly care, and physical exercise. In these scenarios, we demonstrate different features of the system as summarized in Table II. The robot platforms used in these scenarios are NAO and Pepper, which have been proven beneficial in different types of therapies due to their appearances, compactness, and stability e.g. [85]-[87]. At the end of this section, we present a usability evaluation by investigating the therapists' opinions toward the system.

\section{A. Autism therapy: Joint attention}

The first scenario follows a developmental approach of joint attention used in autism therapy intervention studies e.g.
TABLE II

VALIDATED FEATURES IN THREE SCENARIOS

\begin{tabular}{ll}
\hline Scenario & Validated features \\
\hline Autism therapy & $\begin{array}{l}\text { The behavior generation system performance com- } \\
\text { pared with the Wizard-of-Oz }\end{array}$ \\
\hline Elderly care & $\begin{array}{l}\text { The affect system and platform-independent behav- } \\
\text { iors }\end{array}$ \\
\hline Physical exercise & Personalization and affective behaviors \\
\hline
\end{tabular}

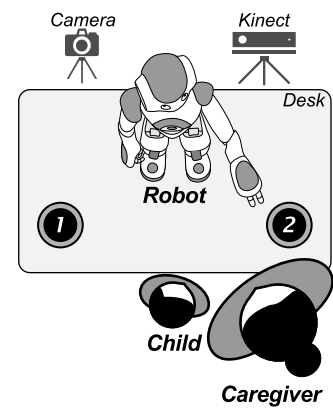

Fig. 9. The experimental setup of the joint attention intervention in autism therapy. A child follows NAO's gaze or pointing to two objects on a desk.

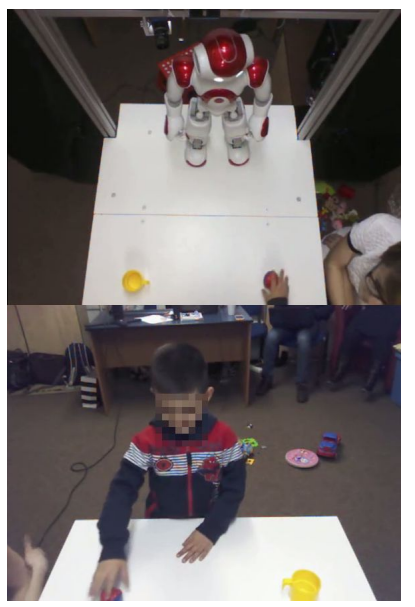

[88], [89]. The idea of this intervention is to increase stepby-step the complexity of the joint attention task from easy (pointing and gaze) to more difficult (gaze only) ones. In this scenario, we compare the performance of the behavior generation system with the WoZ. The affect system was not used to keep the children from being overwhelmed by many social situations.

1) Experimental setup: The setup includes a NAO robot standing on a desk in front of a child who is sitting next to a caregiver as seen in Figure 9. First, the robot introduces the activity to the child. During the interaction, the robot switches its attention to two objects on the desk with different levels of prompts. The robot performs feedback actions according to the child's performance. Finally, the robot says goodbye. 
Wizard of Oz

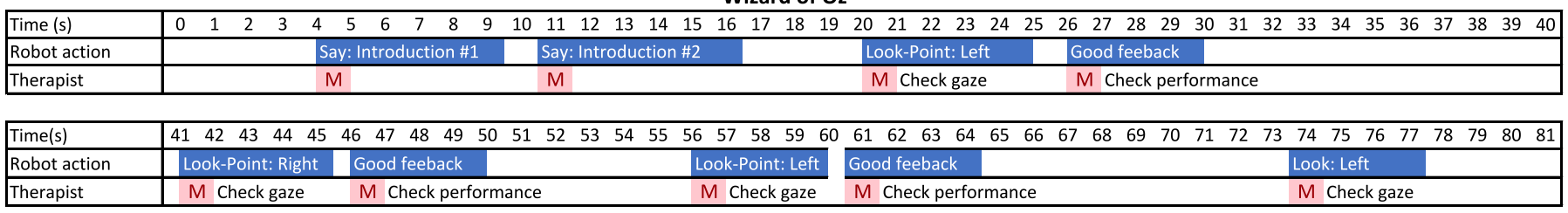

Our proposed system

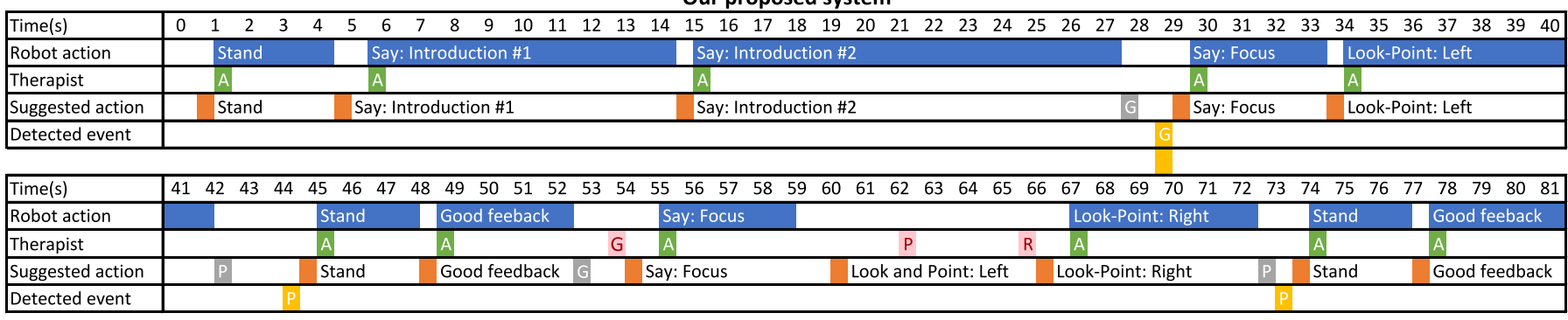

\begin{tabular}{|c|c|c|c|c|c|}
\hline Robot action & Suggested action & A Approve & G Gaze correction & G Waiting for gaze & P Waiting for performance \\
\hline Duration & M Mouse click & P Pause & R Resume & G Gaze detected & Performance detected \\
\hline
\end{tabular}

Fig. 10. Subsets of two interactions using our proposed system and the Wizard of Oz approach. The autonomous behaviors of the robot are comparable with those manually selected by the human therapist. Manual work is therefore reduced.

2) The behavior generation system performance in comparison with the WoZ method: One of the objectives of the system is to reduce manual work for the therapist. In this scenario, we compare the robot autonomous robot behaviors and the behaviors selected by a human therapist in the WoZ in Fig. 10. In the WoZ, the therapist has to constantly observe the interaction events and manually selects proper behaviors by clicking the corresponding buttons. By contrast, our proposed system generates the behaviors autonomously and suggests them to the therapist who has the highest priority of the robot control. In two cases, the executed behaviors are comparable. However, using our proposed system reduces manual work of the therapist during the interaction e.g. looking for proper buttons, detecting user's gaze and performance, keeping track of the interaction flow. The therapist can also access the user's profile including performance history to decide more suitable interacting scenarios for a specific child in the next therapeutic sessions.

\section{B. Elderly care: music therapy}

The second scenario is music therapy for individuals with dementia and cognitive impairment. This activity aims to maintain or improve the user's cognitive attention and enhance the quality of life [90], [91]. This scenario demonstrates the affect system performance and the applicability of our proposed system in another robot platform.

1) Experimental setup: The setup includes a Pepper robot standing in front of a senior user as seen in Fig. 11. First, the robot sends a greeting to the user by referring to his/her name. During the interaction, the robot plays three songs randomly. After a song has been played, the user has to answer the song title using the robot's touchscreen. The robot performs feedback actions according to the user's answers. Finally, the robot says goodbye.
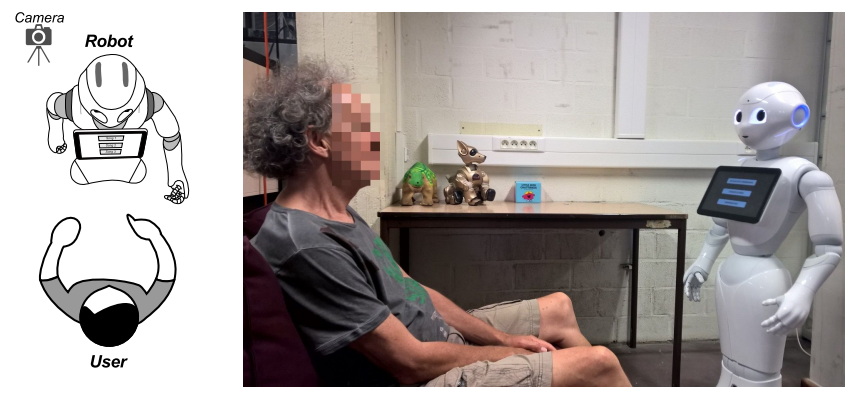

Fig. 11. The experimental setup in the elderly care scenario. A senior user plays a music game with Pepper robot.

2) Platform-independent behavior: Fig. 12 demonstrates a frame-by-frame comparison of a happy behavior expressed on Pepper and NAO. This behavior was used when the user answers the song title correctly. The behavior was coded using a human-based model and the method presented in Subsection IV-F. Although the robots are different in morphologies, the generated behaviors still convey a happy message to the user.

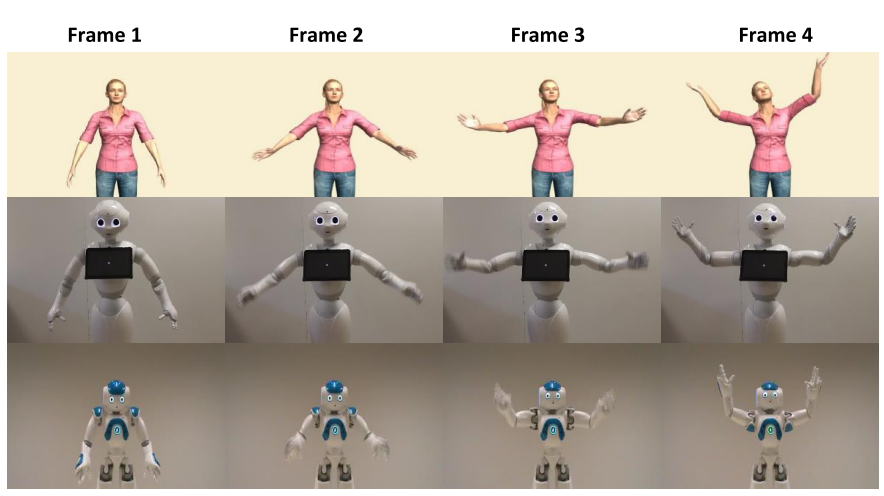

Fig. 12. A happy behavior from a human-based model is expressed on different robot platforms: Pepper and NAO. 
3) Affect system demonstration: The affect system performance can be seen in Fig. 13 in which the affect values evolve differently in two cases of personalities (i.e. extrovert and introvert) under similar stimuli. The affect values in two cases started from the same affect point of joy at the beginning of the interaction. When there was no event, the affect values decayed to their corresponding default values i.e. extrovert case $(0.21,0.0,0.6)$ and introvert case $(-0.21,0.0,-0.6)$ (see Subsection IV-C). When good events (e.g. face detected, correct answer) occurred, the affect values moved to the joy direction. By contrast, they moved to the distress direction if bad events occurred (e.g. incorrect answer). The magnitudes of these moves were influenced by the personalities. Specifically, the introvert character felt emotion more intensely than the extrovert one. The magnitudes of moves in the introvert case were greater than those of the extrovert cases. As a result, the robot behaviors were expressed differently.
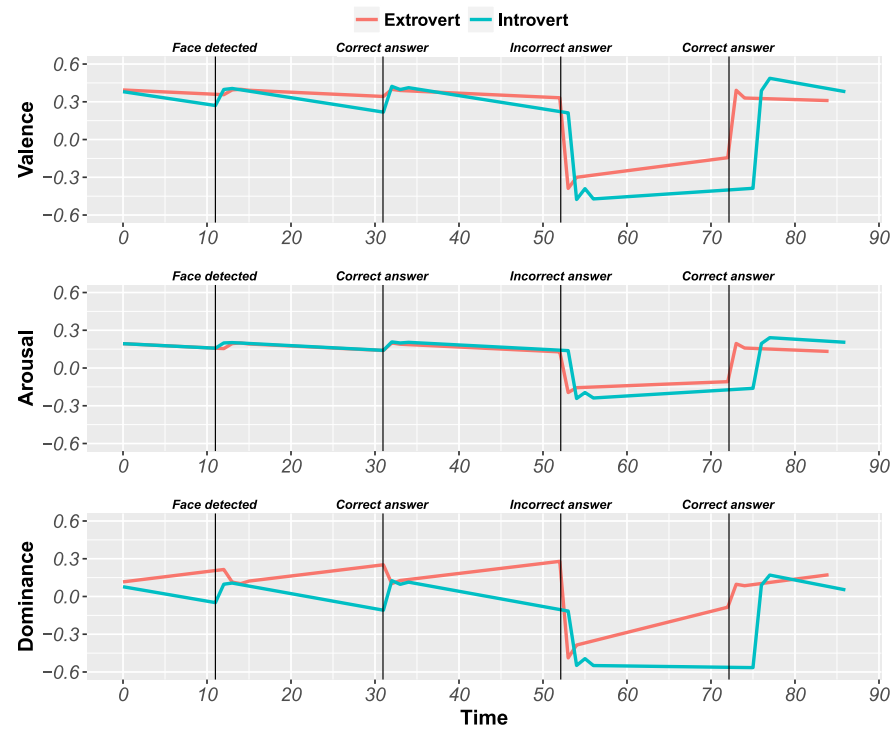

Fig. 13. Evolutions of affect values in two cases of personality: extrovert and introvert, under similar stimuli.

\section{Physical exercise: Shoulder restoration routine}

The third scenario is a shoulder restoration routine physical exercise in which a user performs shoulder stretches and shoulder strengthening exercises. The activity aims to improve shoulder mobility and posture, and pain relief [92]. In this activity, we demonstrate the ability to express personalized affective behaviors.

1) Experimental setup: The setup includes a NAO robot standing on a desk in front of an interacting user as seen in Fig. 14. Light dumbbells are used at some steps of the activity. First, the robot says hello to the user by referring to his/her name, and describes the purpose of the exercise. After that, the robot guides the user through different steps of the exercise. The user receives feedback depending on his/her performance, which also influences the robot's affect system. Finally, the robot says goodbye.
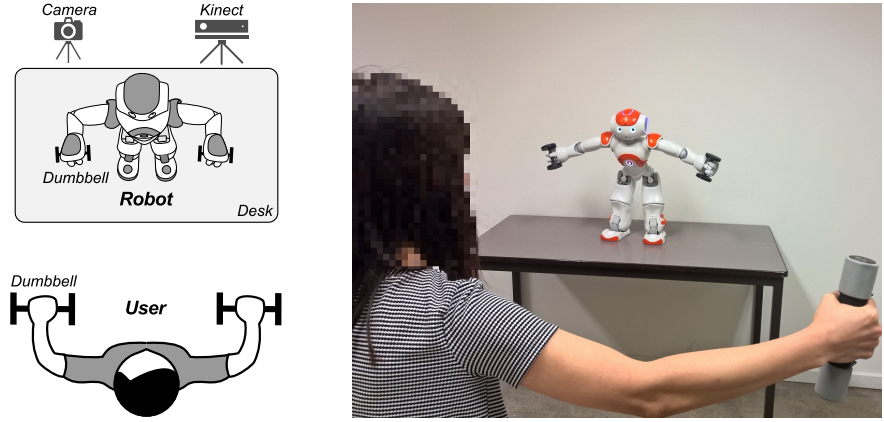

Fig. 14. The experimental setup for the shoulder restoration routine scenario. A user follows NAO's instruction through different exercises.

2) Personalization and affective behaviors validation: We recorded videos from the interactions to perform an online survey investigating the perception of personalization, personality, and affect level. For the personalization perception, we recorded two cases. In the first case, the robot was personalized verbally and nonverbally in which the user manager, reaction and attention subsystem, and the affect system were turned on. The robot, therefore, referred to the user's name and performed affective behaviors. In the second case, the robot was not personalized. We also recorded separate videos to investigate the perception of personality (extrovert and introvert) and affect level (negative, neutral, positive). Manipulation of eye colors was not used to avoid any confounds. Screenshots of videos showing affective behaviors (waving and pointing) can be seen in Fig. 15. All videos are available online ${ }^{1}$.

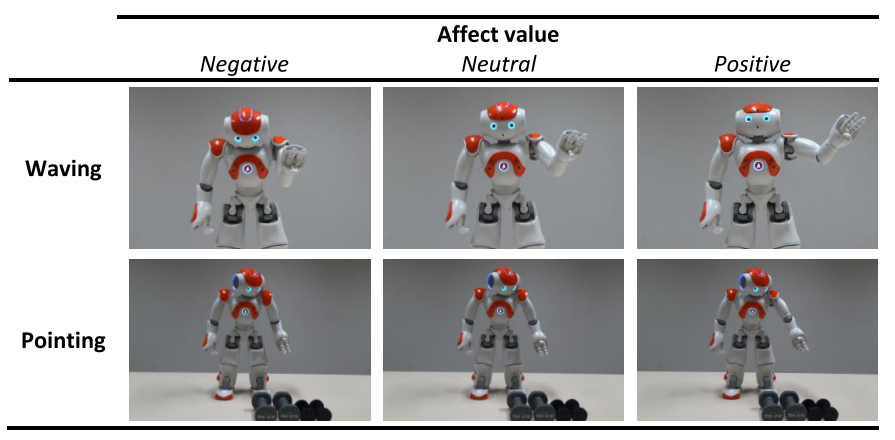

Fig. 15. Screenshots of videos expressing affective behaviors: Waving and Pointing at three affect levels (negative, neutral, positive).

Fourty people (22 men, 18 women) from different backgrounds participated in the survey on Qualtrics. They were recruited through an announcement over the university networks. The age range was from 18 to 37 with the mean age of 26.5 ( $\mathrm{SD}=4.07)$. The videos were presented in a random sequence to avoid order bias. After watching each video, participants were asked to fill in a questionnaire describing the robot's manner. The questionnaire to measure personalization perception was composed of the personalizationrelated items of the Robot Immediacy Questionnaire (RIQ) [93] and Robot Nonverbal Immediacy Questionnaire (RNIQ) [94]. Extroversion-related items of the Big Five Inventory test were used to measure personality perception [95]. And a short

\footnotetext{
${ }^{1}$ hoanglongcao.github.io/research/dorothy\#Videos
} 
version of the PANAS test [96] was used to evaluate the perception of the affect level [97]. The questionnaires use fivepoint Likert scale and are available online ${ }^{2}$.

The survey results are summarized in Fig. 16. In the personalization test, a paired two-tailed t-test reveals a significant difference between the average personalization score of the personalized condition $(\mathrm{M}=3.45,95 \% \mathrm{CI}[3.37,3.53])$ and the non-personalized condition ( $\mathrm{M}=3.13,95 \% \mathrm{CI}[3.05,3.21])$; $\mathrm{t}(39)=5.77, \mathrm{p}<.001$. In the personality expression test, a paired $\mathrm{t}$-test shows that the average extroversion score of the extrovert condition $(\mathrm{M}=2.85,95 \% \mathrm{CI}[2.75,2.95])$ is significantly higher than the introvert condition $(\mathrm{M}=2.66,95 \% \mathrm{CI}[2.55,2.76])$; $\mathrm{t}(39)=2.61, \mathrm{p}<.05$.

A repeated measures analysis using multilevel modelling was conducted to investigate the recognition of affect level. Results show that the total affect level has an influence in the robot behaviors in both waving and pointing. In the waving behavior, there is a significant impact of affective behavior on the affect level score $\left(\mathrm{X}^{2}(2)=71.32, \mathrm{p}<.001\right)$. Posthoc comparisons (Tukey HSD) show that the average score of the positive affect behavior $(\mathrm{M}=3.75,95 \% \mathrm{CI}[3.57,3.92])$ is significantly higher than the neutral affect behavior $(\mathrm{M}=3.08$, 95\% CI [2.94,3.21], $\mathrm{p}<.001)$ and the negative affect behavior $(\mathrm{M}=2.53,95 \% \mathrm{CI}[2.34,2.71], \mathrm{p}<.001)$. The average score of the neutral affect behavior is significantly higher than the negative affect behavior with $\mathrm{p}<.001$.

In the pointing behavior, there is also a significant impact of the pointing behavior on the affect level score $\left(\mathrm{X}^{2}(2)=23.19, \mathrm{p}<.001\right)$. Posthoc comparisons (Tukey HSD) show that the average score of the positive affect behavior $(\mathrm{M}=3.22,95 \% \mathrm{CI}[3.07,3.36])$ is significantly higher than the neutral affect behavior $(\mathrm{M}=2.90,95 \% \mathrm{CI}[2.79,3.00], \mathrm{p}<.001)$ and the negative affect behavior $(\mathrm{M}=2.80,95 \% \mathrm{CI}[2.69,2.91]$, $\mathrm{p}<.001)$. The average score of the neutral affect behavior is not significantly different from the negative affect behavior with $\mathrm{p}$ value of 0.4616 . In this pointing behavior, it is more difficult to recognize affect level since the arm and the head (gaze) directions were constrained.

\section{Usability evaluation}

We conducted a survey on Qualtrics to measure the usability of the system, especially the GUI. We recruited four therapists who have been using our system from three to nine months $(M=6)$. The age range was from 25 to $31(M=26.75)$. The therapists were asked to answer the USE questionnaire consists of 30 five-point Likert scale items divided into four dimensions: Usefulness, Satisfaction, Ease of use, and Ease of learning ${ }^{3}$ [98]. Screenshots of the GUI were used in the questionnaire items to elicit the therapists' memories of their experiences. We also asked open-ended questions to better understand the therapists' opinions. Results showed that the system is useful $(\mathrm{M}=4.06, \mathrm{SD}=0.06)$, satisfying $(\mathrm{M}=3.60$, $\mathrm{SD}=0.68)$, easy to use $(\mathrm{M}=4.23, \mathrm{SD}=0.08)$, and easy to learn $(\mathrm{M}=3.75, \mathrm{SD}=1.09)$. Regarding the therapists' opinions, it is worth noting that although the GUI is composed of many

\footnotetext{
${ }^{2}$ hoanglongcao.github.io/research/dorothy\#Questionnaires

${ }^{3}$ hoanglongcao.github.io/research/dorothy\#GUIEvaluation
}
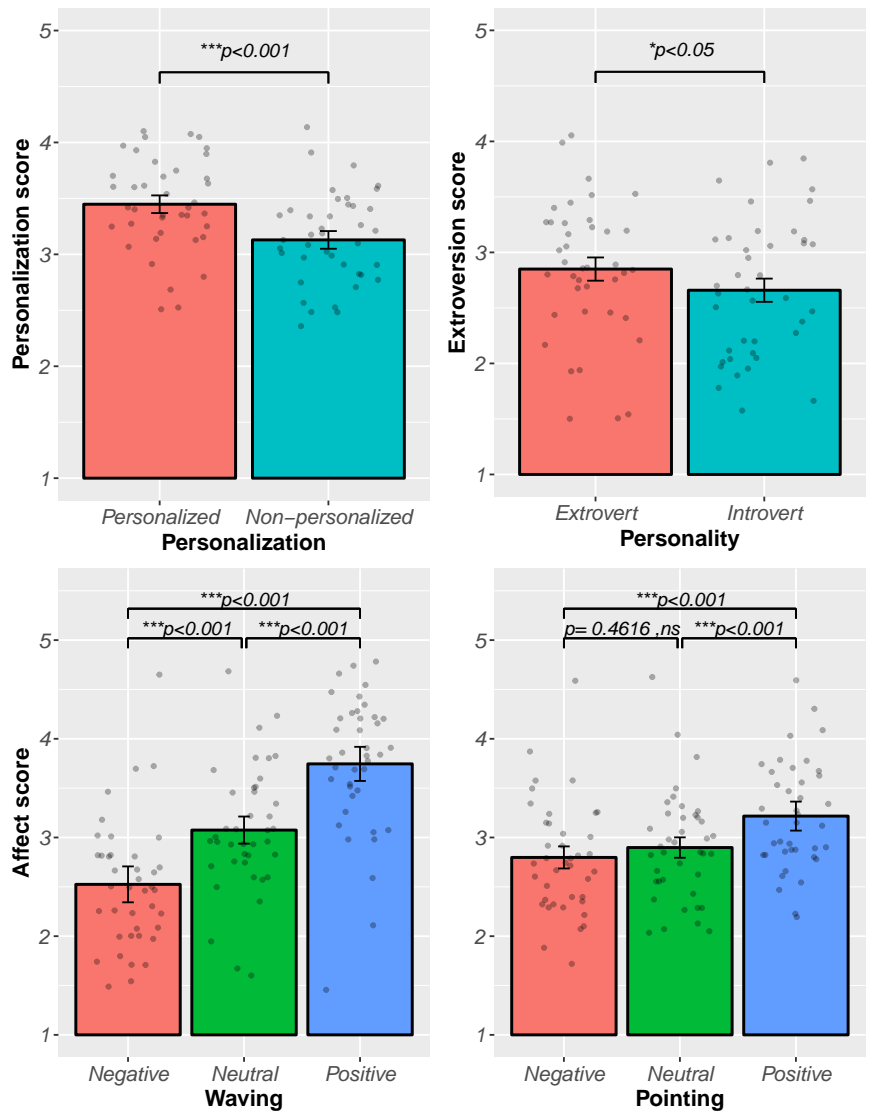

Fig. 16. The survey results. Personalization and affective behaviors were recognized by the participants. Significance is indicated by ${ }^{*} \mathrm{p}<0.05$, ${ }^{*} \mathrm{p}<0.01$, and $* * * \mathrm{p}<0.001$. Error bars show the $95 \%$ Confidence Interval.

group-boxes, the therapists mainly focused on the Scenario Manager and Behavior Supervision during the intervention. However, they suggested having customized interfaces for different applications.

\section{CONCLUSION}

In this paper, we present the development and applications of a platform-independent and personalized behavior control system for social robots in therapies. The system was designed following guidelines drawn from the previous works i.e. multilayer behavior organization, personalization, and modularity. The implementation process follows a component-based software engineering approach using YARP robot developing framework. This allows the developed components to be reusable and replaceable. Robot behaviors were coded for a human-based model and can be transferred to different robot platforms without reprogramming. A human therapist supervises the system operation through a GUI.

The system validation showed that our proposed system is potentially applicable to therapies using different robot platforms. In these scenarios, the system was able to perform tasked-based and social behaviors, which is similar to other behavior control systems in healthcare, e.g., [5], [23], [34], [86], [99]. Personalization, personality, and affective behaviors were recognized as expected. Although previous works also 
implemented these features (e.g., [13], [46], [73], [74], [100]), our system is platform-independent and able to generate the robot behaviors for different robot platforms. To our knowledge, there is a very limited number of studies considering platform-independence, especially in healthcare domain [6], [7], [12]. The usability evaluation investigating the therapists' opinions showed that the system is useful, satisfying, easy to use, and easy to learn.

With the component-based and platform-independent approaches, our system architecture is expected to serve as a basic framework for researchers to customize and expand the system for their targeted healthcare applications. We are currently working on larger scale experiments using the system e.g. investigating the children's performance between childtherapist interaction and child-robot interaction, the effect of personalization on the user's performance, comparing the effect of user-robot personality matching in different tasks.

\section{ACKNOWLEDGMENT}

The work leading to these results has received funding from the European Commission 7th Framework Program as a part of the project DREAM grant no. 611391. The authors would like to thank the therapists from Vrije Universiteit Brussel (Belgium), Kinetiek BVBA (Belgium), HASfit (USA), and Babes-Bolyai University (Romania) for their valuable inputs and feedback on the system development and validation. Other acknowledgements are to SoftBank Robotics Europe (France) and DLR (Germany).

\section{REFERENCES}

[1] A. Tapus, M. J. Mataric, and B. Scasselati, "Socially assistive robotics [grand challenges of robotics]," Robotics \& Automation Magazine, IEEE, vol. 14, no. 1, pp. 35-42, 2007.

[2] C. Breazeal, "Social robots for health applications," in Engineering in Medicine and Biology Society, EMBC, 2011 Annual International Conference of the IEEE. IEEE, 2011, pp. 5368-5371.

[3] M. J. Matarić and B. Scassellati, "Socially assistive robotics," in Springer Handbook of Robotics. Springer, 2016, pp. 1973-1994.

[4] T. Belpaeme, P. E. Baxter, R. Read, R. Wood, H. Cuayáhuitl, B. Kiefer, S. Racioppa, I. Kruijff-Korbayová, G. Athanasopoulos, V. Enescu, et al., "Multimodal child-robot interaction: Building social bonds," Journal of Human-Robot Interaction, vol. 1, no. 2, pp. 33-53, 2012.

[5] J. Fasola and M. Mataric, "A socially assistive robot exercise coach for the elderly," Journal of Human-Robot Interaction, vol. 2, no. 2, pp. 3-32, 2013.

[6] H.-L. Cao, P. G. Esteban, A. De Beir, R. Simut, G. Van de Perre, D. Lefeber, and B. Vanderborght, "A survey on behavior control architectures for social robots in healthcare interventions," International Journal of Humanoid Robotics, pp. 1-21, 2017.

[7] P. G. Esteban, P. Baxter, T. Belpaeme, E. Billing, H. Cai, H.-L. Cao, M. Coeckelbergh, C. Costescu, D. David, A. De Beir, et al., "How to build a supervised autonomous system for robot-enhanced therapy for children with autism spectrum disorder," Paladyn: journal of behavioral robotics, pp. 1-22, 2017.

[8] T. K. Landauer, "Psychology as a mother of invention," ACM SIGCHI Bulletin, vol. 18, no. 4, pp. 333-335, 1987.

[9] J. Wilson and D. Rosenberg, "Rapid prototyping for user interface design," 1988.

[10] L. D. Riek, "Robotics technology in mental health care," Artificial Intelligence in Behavioral and Mental Health Care, p. 185, 2015.

[11] S. M. Rabbitt, A. E. Kazdin, and B. Scassellati, "Integrating socially assistive robotics into mental healthcare interventions: Applications and recommendations for expanded use," Clinical psychology review, vol. 35, pp. 35-46, 2015.
[12] S. Thill, C. A. Pop, T. Belpaeme, T. Ziemke, and B. Vanderborght, "Robot-assisted therapy for autism spectrum disorders with (partially) autonomous control: Challenges and outlook," Paladyn, Journal of Behavioral Robotics, vol. 3, no. 4, pp. 209-217, 2012.

[13] P. Baxter, E. Ashurst, R. Read, J. Kennedy, and T. Belpaeme, "Robot education peers in a situated primary school study: Personalisation promotes child learning," PLoS One, vol. 12, no. 5, p. e0178126, 2017.

[14] K. Dautenhahn, "Robots we like to live with?!-a developmental perspective on a personalized, life-long robot companion," in Robot and human interactive communication, 2004. ROMAN 2004. 13th IEEE International Workshop on. IEEE, 2004, pp. 17-22.

[15] M. K. Lee, J. Forlizzi, S. Kiesler, P. Rybski, J. Antanitis, and S. Savetsila, "Personalization in hri: A longitudinal field experiment," in Human-Robot Interaction (HRI), 2012 7th ACM/IEEE International Conference on. IEEE, 2012, pp. 319-326.

[16] I. Nahum-Shani, E. B. Hekler, and D. Spruijt-Metz, "Building health behavior models to guide the development of just-in-time adaptive interventions: A pragmatic framework." Health Psychology, vol. 34, no. S, p. 1209, 2015.

[17] S. Rossi, F. Ferland, and A. Tapus, "User profiling and behavioral adaptation for hri: A survey," Pattern Recognition Letters, 2017.

[18] M. Coeckelbergh, C. Pop, R. Simut, A. Peca, S. Pintea, D. David, and B. Vanderborght, "A survey of expectations about the role of robots in robot-assisted therapy for children with asd: Ethical acceptability, trust, sociability, appearance, and attachment," Science and engineering ethics, pp. 1-19, 2015.

[19] D. Feil-Seifer and M. J. Mataric, "Dry your eyes: examining the roles of robots for childcare applications," Interaction Studies, vol. 11, no. 2, p. 208, 2010.

[20] N. Giullian, D. Ricks, A. Atherton, M. Colton, M. Goodrich, and B. Brinton, "Detailed requirements for robots in autism therapy," in Systems Man and Cybernetics (SMC), 2010 IEEE International Conference on. IEEE, 2010, pp. 2595-2602.

[21] I. Zubrycki and G. Granosik, "Understanding therapists needs and attitudes towards robotic support. the roboterapia project," International Journal of Social Robotics, vol. 8, no. 4, pp. 553-563, 2016.

[22] M. Martin-Ortiz, M.-G. Kim, and E. I. Barakova, "Mobile application for executing therapies with robots," in International Work-Conference on Artificial Neural Networks. Springer, 2017, pp. 82-92.

[23] H.-L. Cao, P. G. Esteban, R. Simut, G. Van de Perre, D. Lefeber, B. Vanderborght, et al., "A collaborative homeostatic-based behavior controller for social robots in human-robot interaction experiments," International Journal of Social Robotics, pp. 1-16.

[24] K. Dautenhahn and C. L. Nehaniv, "The correspondence problem," in Imitation in Animals and Artifacts, MIT Press. MIT Press, 2002.

[25] A. Alissandrakis, C. L. Nehaniv, and K. Dautenhahn, "Imitation with alice: Learning to imitate corresponding actions across dissimilar embodiments," IEEE Transactions on Systems, Man, and CyberneticsPart A: Systems and Humans, vol. 32, no. 4, pp. 482-496, 2002.

[26] Q. A. Le, S. Hanoune, and C. Pelachaud, "Design and implementation of an expressive gesture model for a humanoid robot," in Humanoid Robots (Humanoids), 2011 11th IEEE-RAS International Conference on. IEEE, 2011, pp. 134-140.

[27] M. Salem, S. Kopp, I. Wachsmuth, and F. Joublin, "Generating multimodal robot behavior based on a virtual agent framework," in Proceedings of the ICRA 2010 Workshop on Interactive Communication for Autonomous Intelligent Robots (ICAIR), 2010.

[28] G. Van de Perre, A. De Beir, H.-L. Cao, P. G. Esteban, D. Lefeber, and B. Vanderborght, "Reaching and pointing gestures calculated by a generic gesture system for social robots," Robotics and Autonomous Systems, vol. 83, pp. 32-43, 2016.

[29] G. Van de Perre, M. Van Damme, D. Lefeber, and B. Vanderborght, "Development of a generic method to generate upper-body emotional expressions for different social robots," Advanced Robotics, vol. 29, no. 9, pp. 597-609, 2015.

[30] D. Brugali and A. Shakhimardanov, "Component-based robotic engineering (part ii)," IEEE Robotics \& Automation Magazine, vol. 17, no. 1, pp. 100-112, 2010.

[31] D. Brugali and P. Scandurra, "Component-based robotic engineering (part i)[tutorial]," IEEE Robotics \& Automation Magazine, vol. 16, no. 4, pp. 84-96, 2009.

[32] D. Vernon, E. Billing, P. Hemeren, S. Thill, and T. Ziemke, "An architecture-oriented approach to system integration in collaborative robotics research projects: An experience report," Journal of Software Engineering for Robotics, vol. 6, no. 1, pp. 15-32, 2015.

[33] M. Y. Jung, M. Balicki, A. Deguet, R. H. Taylor, and P. Kazanzides, "Lessons learned from the development of component-based medical 
robot systems," Journal of Software Engineering for Robotics, vol. 5, no. 2, pp. 25-41, 2014.

[34] D. Feil-Seifer and M. J. Mataric, "B3ia: A control architecture for autonomous robot-assisted behavior intervention for children with autism spectrum disorders," in Robot and Human Interactive Communication, 2008. RO-MAN 2008. The 17th IEEE International Symposium on. IEEE, 2008, pp. 328-333.

[35] H.-L. Cao, C. Pop, R. Simut, R. Furnémont, A. De Beir, G. Van de Perre, P. G. Esteban, D. Lefeber, and B. Vanderborght, "Probolino: A portable low-cost social device for home-based autism therapy," in Social Robotics. Springer, 2015, pp. 93-102.

[36] J. Wainer, K. Dautenhahn, B. Robins, and F. Amirabdollahian, "A pilot study with a novel setup for collaborative play of the humanoid robot kaspar with children with autism," International Journal of Social Robotics, vol. 6, no. 1, pp. 45-65, 2014.

[37] R. Simut, J. Vanderfaeillie, A. Peca, G. V. de Perre, and B. Vanderborght, "Exploring joint attention: 9-18 months old typically developing infants in responding and initiating joint attention with a human partner and with the social robot Keepon," Journal of Autism and Developmental Disorders, submitted for the Special Issue: Robotics \& Technology and Computer Assistance, 2014.

[38] R. C. Arkin, M. Fujita, T. Takagi, and R. Hasegawa, "An ethological and emotional basis for human-robot interaction," Robotics and Autonomous Systems, vol. 42, no. 3, pp. 191-201, 2003.

[39] D. McColl, W.-Y. G. Louie, and G. Nejat, "Brian 2.1: A socially assistive robot for the elderly and cognitively impaired," Robotics \& Automation Magazine, IEEE, vol. 20, no. 1, pp. 74-83, 2013.

[40] S. Calinon and A. Billard, "Pda interface for humanoid robots," in Proceedings of the IEEE International Conference on Humanoid Robots (Humanoids), no. LSA3-CONF-2003-002, 2003.

[41] G. Pioggia, M. Sica, M. Ferro, R. Igliozzi, F. Muratori, A. Ahluwalia, and D. D. Rossi, "Human-robot interaction in autism: Face, an androidbased social therapy," in Robot and Human interactive Communication, 2007. RO-MAN 2007. The 16th IEEE International Symposium on. IEEE, 2007, pp. 605-612.

[42] N. Lazzeri, D. Mazzei, A. Zaraki, and D. De Rossi, "Towards a believable social robot," in Biomimetic and Biohybrid Systems. Springer, 2013, pp. 393-395.

[43] J. Saldien, B. Vanderborght, K. Goris, M. Van Damme, and D. Lefeber, "A motion system for social and animated robots," International Journal of Advanced Robotic Systems, vol. 11, 2014.

[44] D. David, S.-A. Matu, and O. A. David, "Robot-based psychotherapy: Concepts development, state of the art, and new directions," International Journal of Cognitive Therapy, vol. 7, no. 2, pp. 192-210, 2014.

[45] C. A. Pop, S. Pintea, B. Vanderborght, and D. O. David, "Enhancing play skills, engagement and social skills in a play task in asd children by using robot-based interventions. a pilot study," Interaction Studies, vol. 15 , no. 2, pp. 292-320, 2014.

[46] A. Tapus, C. Ţăpuş, and M. J. Matarić, "User-robot personality matching and assistive robot behavior adaptation for post-stroke rehabilitation therapy," Intelligent Service Robotics, vol. 1, no. 2, pp. 169-183, 2008.

[47] C. M. Stanton, P. H. Kahn Jr, R. L. Severson, J. H. Ruckert, and B. T. Gill, "Robotic animals might aid in the social development of children with autism," in Human-Robot Interaction (HRI), 2008 3rd ACM/IEEE International Conference on. IEEE, 2008, pp. 271-278.

[48] D. A. Norman, A. Ortony, and D. M. Russell, "Affect and machine design: Lessons for the development of autonomous machines," IBM Systems Journal, vol. 42, no. 1, pp. 38-44, 2003.

[49] A. Ortony, D. Norman, and W. Revelle, "Affect and proto-affect in effective functioning. who needs emotions," The Brain Meets the Robot, 2005.

[50] A. Sloman and B. Logan, "Evolvable architectures for human-like minds," in Affective Minds, 2000.

[51] A. Sloman et al., "Beyond shallow models of emotion," Cognitive Processing: International Quarterly of Cognitive Science, vol. 2, no. 1, pp. 177-198, 2001.

[52] A. B. Karami, K. Sehaba, and B. Encelle, "Adaptive artificial companions learning from users feedback," Adaptive Behavior, vol. 24, no. 2, pp. 69-86, 2016.

[53] A. Aly and A. Tapus, "Towards an intelligent system for generating an adapted verbal and nonverbal combined behavior in human-robot interaction," Autonomous Robots, vol. 39, no. 1, 2015.

[54] M. Joosse, M. Lohse, J. G. Pérez, and V. Evers, "What you do is who you are: The role of task context in perceived social robot personality," in Robotics and automation (ICRA), 2013 IEEE international conference on. IEEE, 2013, pp. 2134-2139.
[55] S. Woods, K. Dautenhahn, C. Kaouri, R. te Boekhorst, K. L. Koay, and M. L. Walters, "Are robots like people?: Relationships between participant and robot personality traits in human-robot interaction studies," Interaction Studies, vol. 8, no. 2, pp. 281-305, 2007.

[56] L. R. Goldberg, "An alternative" description of personality": the bigfive factor structure." Journal of personality and social psychology, vol. 59, no. 6, p. 1216, 1990.

[57] R. R. McCrae and O. P. John, "An introduction to the five-factor model and its applications," Journal of personality, vol. 60, no. 2, pp. 175215, 1992 .

[58] A. Mehrabian, "Pleasure-arousal-dominance: A general framework for describing and measuring individual differences in temperament," Current Psychology, vol. 14, no. 4, pp. 261-292, 1996.

[59] A. Ortony, G. L. Clore, and A. Collins, The cognitive structure of emotions. Cambridge university press, 1990.

[60] A. Egges, S. Kshirsagar, and N. Magnenat-Thalmann, "A model for personality and emotion simulation," in International Conference on Knowledge-Based and Intelligent Information and Engineering Systems. Springer, 2003, pp. 453-461.

[61] P. Gebhard, "Alma: a layered model of affect," in Proceedings of the fourth international joint conference on Autonomous agents and multiagent systems. ACM, 2005, pp. 29-36.

[62] A. Mehrabian, "Analysis of the big-five personality factors in terms of the pad temperament model," Australian Journal of Psychology, vol. 48, no. 2, pp. 86-92, 1996.

[63] T. Doce, J. Dias, R. Prada, and A. Paiva, "Creating individual agents through personality traits." in IVA. Springer, 2010, pp. 257-264.

[64] P. Gómez Esteban, H.-L. Cao, A. De Beir, G. Van de Perre, D. Lefeber, and B. Vanderborght, "A multilayer reactive system for robots interacting with children with autism," 2016.

[65] J. Shim and R. C. Arkin, "An intervening ethical governor for a robot mediator in patient-caregiver relationships," in A World with Robots. Springer, 2017, pp. 77-91.

[66] E. I. Barakova, J. C. Gillesen, B. E. Huskens, and T. Lourens, "Enduser programming architecture facilitates the uptake of robots in social therapies," Robotics and Autonomous Systems, vol. 61, no. 7, pp. 704713, 2013.

[67] H.-L. Cao, A. De Beir, P. G. Esteban, R. Simut, G. Van de Perre, D. Lefeber, and B. Vanderborght, "An end-user interface to generate homeostatic behavior for nao robot in robot-assisted social therapies," in International Work-Conference on Artificial Neural Networks. Springer, 2017, pp. 609-619.

[68] I. Zubrycki, M. Kolesiński, and G. Granosik, "Graphical programming interface for enabling non-technical professionals to program robots and internet-of-things devices," in International Work-Conference on Artificial Neural Networks. Springer, 2017, pp. 620-631.

[69] E. Senft, P. Baxter, J. Kennedy, and T. Belpaeme, "SPARC: Supervised progressively autonomous robot competencies," in International Conference on Social Robotics. Springer, 2015, pp. 603-612.

[70] E. Senft, P. Baxter, J. Kennedy, S. Lemaignan, and T. Belpaeme, "Supervised autonomy for online learning in human-robot interaction," Pattern Recognition Letters, 2017.

[71] B. Siciliano, L. Sciavicco, L. Villani, and G. Oriolo, Robotics: modelling, planning and control. Springer Science \& Business Media, 2010.

[72] U. M. Ascher and L. R. Petzold, Computer methods for ordinary differential equations and differential-algebraic equations. Siam, 1998, vol. 61.

[73] J. Xu, J. Broekens, K. Hindriks, and M. A. Neerincx, "Mood expression through parameterized functional behavior of robots," in 2013 IEEE RO-MAN, 2013, pp. 533-540.

[74] — - "Mood contagion of robot body language in human robot interaction," Autonomous Agents and Multi-Agent Systems, vol. 29, no. 6, pp. 1216-1248, 2015.

[75] R. A. Lippa and J. K. Dietz, “The relation of gender, personality, and intelligence to judges' accuracy in judging strangers' personality from brief video segments," Journal of Nonverbal Behavior, vol. 24, no. 1, pp. 25-43, 2000.

[76] K. M. Lee, W. Peng, S.-A. Jin, and C. Yan, "Can robots manifest personality?: An empirical test of personality recognition, social responses, and social presence in human-robot interaction," Journal of communication, vol. 56, no. 4, pp. 754-772, 2006.

[77] K. Amaya, A. Bruderlin, and T. Calvert, "Emotion from motion," in Graphics interface, vol. 96. Toronto, Canada, 1996, pp. 222-229.

[78] C. Pelachaud, "Studies on gesture expressivity for a virtual agent," Speech Communication, vol. 51, no. 7, pp. 630-639, 2009. 
[79] A. Yamaguchi, Y. Yano, S. Doki, and S. Okuma, "A study of emotional motion description by motion modification rules using adjectival expressions," in Systems, Man and Cybernetics, 2006. SMC'06. IEEE International Conference on, vol. 4. IEEE, 2006, pp. 2837-2842.

[80] C. Hu, M. A. Walker, M. Neff, and J. E. F. Tree, "Storytelling agents with personality and adaptivity," in International Conference on Intelligent Virtual Agents. Springer, 2015, pp. 181-193.

[81] H. Kim, S. S. Kwak, and M. Kim, "Personality design of sociable robots by control of gesture design factors," in Robot and Human Interactive Communication, 2008. RO-MAN 2008. The 17th IEEE International Symposium on. IEEE, 2008, pp. 494-499.

[82] D. Vernon, E. Billing, P. Hemeren, S. Thill, and T. Ziemke, "An architecture-oriented approach to system integration in collaborative robotics research projects: An experience report," Journal of Software Engineering for Robotics, vol. 6, no. 1, pp. 15-32, 2015.

[83] P. Fitzpatrick, E. Ceseracciu, D. Domenichelli, A. Paikan, G. Metta, and L. Natale, "A middle way for robotics middleware," 2014.

[84] G. Metta, P. Fitzpatrick, and L. Natale, "Yarp: yet another robot platform," International Journal of Advanced Robotic Systems, vol. 3, no. 1 , p. 8, 2006.

[85] D. Gouaillier, V. Hugel, P. Blazevic, C. Kilner, J. Monceaux, P. Lafourcade, B. Marnier, J. Serre, and B. Maisonnier, "Mechatronic design of NAO humanoid," in Robotics and Automation, 2009. ICRA'09. IEEE International Conference on. IEEE, 2009, pp. 769-774.

[86] B. Görer, A. A. Salah, and H. L. Akın, "An autonomous robotic exercise tutor for elderly people," Autonomous Robots, vol. 41, no. 3, pp. 657-678, 2017.

[87] A. Tapus, A. Peca, A. Aly, C. Pop, L. Jisa, S. Pintea, A. S. Rusu, and D. O. David, "Children with autism social engagement in interaction with nao, an imitative robot-a series of single case experiments," Interaction studies, vol. 13, no. 3, pp. 315-347, 2012.

[88] I. Schietecatte, H. Roeyers, and P. Warreyn, "Exploring the nature of joint attention impairments in young children with autism spectrum disorder: Associated social and cognitive skills," Journal of autism and developmental disorders, vol. 42, no. 1, pp. 1-12, 2012.

[89] C. Whalen and L. Schreibman, "Joint attention training for children with autism using behavior modification procedures," Journal of Child Psychology and Psychiatry, vol. 44, no. 3, pp. 456-468, 2003.

[90] A. A. Clair and A. G. Ebberts, "The effects of music therapy on interactions between family caregivers and their care receivers with late stage dementia," Journal of Music Therapy, vol. 34, no. 3, pp. 148-164, 1997.

[91] A. Tapus, "Improving the quality of life of people with dementia through the use of socially assistive robots," in Advanced Technologies for Enhanced Quality of Life, 2009. AT-EQUAL'09. IEEE, 2009, pp. $81-86$.

[92] Hasfit, "20 min shoulder stretches \& strengthening for pain relief," 01 2017. [Online]. Available: http://hasfit.com/workouts/home/senior/ shoulder-stretches-strengthening-for-pain-relief/

[93] J. Kennedy, P. Baxter, E. Senft, and T. Belpaeme, "Higher nonverbal immediacy leads to greater learning gains in child-robot tutoring interactions," in International conference on social robotics. Springer, 2015, pp. 327-336.

[94] — - "Social robot tutoring for child second language learning," in Human-Robot Interaction (HRI), 2016 11th ACM/IEEE International Conference on. IEEE, 2016, pp. 231-238.

[95] G. Saucier, "Mini-markers: A brief version of goldberg's unipolar bigfive markers," Journal of personality assessment, vol. 63, no. 3, pp. 506-516, 1994.

[96] D. Watson, L. A. Clark, and A. Tellegen, "Development and validation of brief measures of positive and negative affect: the panas scales." Journal of personality and social psychology, vol. 54, no. 6, p. 1063, 1988.

[97] S. Park, L. Moshkina, and R. C. Arkin, "Recognizing nonverbal affective behavior in humanoid robots," in Intelligent Autonomous Systems, vol. 11, 2010, p. 12.

[98] A. M. Lund, "Measuring usability with the use questionnaire12," Usability interface, vol. 8, no. 2, pp. 3-6, 2001.

[99] D. Feil-Seifer and M. J. Matarić, "Toward socially assistive robotics for augmenting interventions for children with autism spectrum disorders," in Experimental robotics. Springer, 2009, pp. 201-210.
[100] L. Moshkina, S. Park, R. C. Arkin, J. K. Lee, and H. Jung, "Tame: Time-varying affective response for humanoid robots," International Journal of Social Robotics, vol. 3, no. 3, pp. 207-221, 2011.

Hoang-Long Cao received his B.Eng. in Mechatronics from Can Tho University in 2010, and M.Sc. in Electromechanical engineering from the Vrije Universiteit Brussel and Université Libre de Bruxelles in 2013. From September 2013, he has been working as a Ph.D. researcher at the Multibody Mechanics and Robotics research group, Vrije Universiteit Brussel, Belgium. His research interest is social robotics. Currently he is involved in DREAM project for the development of robot-enhanced therapy for children with autism spectrum disorders.

Greet Van de Perre studied Mechanical Engineering at the Vrije Universiteit Brussel. After graduating in 2011, she started her Ph.D. at the Robotics and Multibody Mechanics Research Group, funded by an FWO fellowship. Her research focuses on the development of a generic gesture system for social robots and the design of an actuated upper-torso for the social robot Probo.

James Kennedy received his Ph.D. from Plymouth University, U.K. in 2017 for his work using social robots to tutor children. During his $\mathrm{PhD}$, he worked as a Research Assistant on the EU-funded DREAM (Development of RobotEnhanced therapy for children with AutisM spectrum disorders) project, and collaborated with the ALIZ-E (Adaptive Strategies for Sustainable Long-Term Social Interaction), and L2TOR (Second Language Tutoring using Social Robots) projects. In 2016, James was a Lab Associate with Disney Research, Pittsburgh. His research interests lie in Human-Robot Interaction and Socially Intelligent Agents.

Emmanuel Senft received his Master of Science from EPFL in 2013 in Robotic and Autonomous Systems. He is a $\mathrm{PhD}$ student in Human Robot Interaction at Plymouth University since 2014. His interests are mainly interactive machine learning and social robotic and especially how to use humans to teach robots how to interact with other humans. He is involved in the EU FP7 DREAM project aiming at developing new robot enhanced therapies for children with autism.

Pablo Gómez Esteban received, in March 2014 from the Rey Juan Carlos University of Madrid, a Ph.D. degree for his thesis entitled "Cooperation and Competition in Emotional Robot Societies" with highest distinction. $\mathrm{He}$ is currently a post-doctoral researcher working at Vrije Universiteit Brussel in Belgium. His research was addressed towards decision making processes within competitive and cooperative environments; the impact that affective factors have in such processes; and designing and implementing those models to be used in non-expensive platforms within domestic environments. But his research interests also lie on cognitive architectures for social robots and human-robot interaction. He is the author or co-author of several chapters in books and published articles in international journals and conferences.

Albert De Beir received in 2014 his Master degree in electromechanical engineering from the Vrije Universiteit Brussel and in 2016 his Master degree in Psychology from the Université Libre de Bruxelles. His research interest focus on the development of artificial intelligence for social robots. 
Ramona Simut received her M.S. degrees from Babes-Bolyai University in 2011. From 2008 to 2011 she worked as a psychologist at the Research Department of Autism Transylvania Association on the project of Using the social robot Probo in therapy for children with ASD. From September 2011 to present she is a Ph.D. student with the research focus on the clinical use of social robots for ASD children at the Clinical and Life Span Psychology Department of Vrije Universiteit Brussel. Her research interests include developing robot assisted therapies for children with autism, perception of the child-robot interaction, responses to robots of children with ASD.

Tony Belpaeme received his Ph.D. in Computer Science from the Vrije Universiteit Brussel in 2002 and is currently Professor in Robotics and Cognitive Systems at Plymouth University (UK) where he leads a research lab in the Centre for Robotics and Neural Systems. Starting from the premise that cognition is rooted in social interaction, Belpaeme and team try to further the science and technology behind artificial intelligence and social robots. This results in a spectrum of fundings, from theoretical insights to practical applications. He coordinated the FP7 ALIZ-E project, and collaborated on the ROBOT-ERA, DREAM and ITALK projects. He is currently the coordinator of the H2020 L2TOR project.
Dirk Lefeber received a degree in Civil Engineering in 1979 and a Ph.D. in Applied Sciences in 1986 from the Vrije Universiteit Brussel. Currently, he is full professor, former head of the department of Mechanical Engineering and head of the Robotics and Multibody Mechanics Research Group, Vrije Universiteit Brussel, which he founded in 1990. The research interests of the group are new actuators with adaptable compliance, dynamically balanced robots, robot assistants, rehabilitation robotics and multibody dynamics, all in the context of physical and cognitive human robot interaction with emphasis on improving the quality of life of human.

Bram Vanderborght focused his Ph.D. research on the use of adaptable compliance of pneumatic artificial muscles in the dynamically balanced biped Lucy. In May-June 2006, he performed research on the humanoids robot HRP-2 at the Joint Japanese/French Robotics Laboratory (JRL) in AIST, Tsukuba (Japan) in the research 'Dynamically stepping over large obstacles by the humanoid robot HRP-2'. From October 2007-April 2010 he worked as post-doc researcher at the Italian Institute of Technology in Genova (Italy) on the humanoid robot iCub and compliant actuation. Since October 2009, he is appointed as professor at the Vrije Universiteit Brussel. He has an ERR starting grant on SPEA actuation concept. He is member of the Young Academy of the Royal Flemish Academy of Belgium for Science and the Arts. His research interests include cognitive and physical human robot interaction, social robots, humanoids, robot assisted therapy and rehabilitation/assistive robotics. He is current EiC of IEEE Robotics \& Automation Magazine. 